\title{
Retraction
}

\section{Retracted: The Immune System in Hepatocellular Carcinoma and Potential New Immunotherapeutic Strategies}

\author{
BioMed Research International \\ Received 16 March 2016; Accepted 16 March 2016 \\ Copyright (C) 2016 BioMed Research International. This is an open access article distributed under the Creative Commons \\ Attribution License, which permits unrestricted use, distribution, and reproduction in any medium, provided the original work is \\ properly cited.
}

The article titled "The Immune System in Hepatocellular Carcinoma and Potential New Immunotherapeutic Strategies" [1] has been retracted as it was found to contain a substantial amount of material, without referencing, from the following published article: "Potential of immunotherapy for hepatocellular carcinoma," by Ekaterina Breous and Robert Thimme, in Journal of Hepatology. The author list has been corrected to remove Salvatore Gruttadauria, Isidoro Di Carlo, Adriana Toro and Alessandra Mangia, who were not involved in the preparation or submission of this article and should not have been listed as authors. The first author, Gaetano Bertino, accepts full responsibility and apologizes to the journal and the other authors. The rest of the authors are not culpable in this plagiarism and are victims of this action.

\section{References}

[1] G. Bertino, S. Demma, A. Ardiri et al., "The immune system in hepatocellular carcinoma and potential new immunotherapeutic strategies," BioMed Research International, vol. 2015, Article ID 731469, 12 pages, 2015. 


\title{
The Immune System in Hepatocellular Carcinoma and Potential New Immunotherapeutic Strategies
}

\author{
Gaetano Bertino, ${ }^{1}$ Shirin Demma, ${ }^{1}$ Annalisa Ardiri, ${ }^{1}$ Maria Proiti, ${ }^{1}$ Giulia Malaguarnera, ${ }^{2}$ \\ Nicoletta Bertino, ${ }^{3,4}$ Mariano Malaguarnera, ${ }^{5}$ and Michele Malaguarnera ${ }^{2,6}$ \\ ${ }^{1}$ Department of Medical and Pediatric Sciences, Hepatology Unit, University of Catania, Policlinic, Via S. Sofia No. 78, \\ 95123 Catania, Italy \\ ${ }^{2}$ Research Centre "The Great Senescence", University of Catania, Cannizzaro Hospital, Via Messina No. 829, 95100 Catania, Italy \\ ${ }^{3}$ Faculty of Pharmacy, University of Catania, Viale Andrea Doria No. 6, 95123 Catania, Italy \\ ${ }^{4}$ Faculty of Pharmacy, University of Catania, University of Catania Policlinic, Via S. Sofia No. 78, 95123 Catania, Italy \\ ${ }^{5}$ Department of Medical and Pediatric Science, Research Centre "The Great Senescence", University of Catania, \\ Cannizzaro Hospital, Via Messina No. 829, 95100 Catania, Italy \\ ${ }^{6}$ International Ph.D. Program in Neuropharmacology, University of Catania, Cannizzaro Hospital, Via Messina No. 829, \\ 95100 Catania, Italy
}

Correspondence should be addressed to Gaetano Bertino; gaetanobertinounict@libero.it

Received 25 July 2014; Revised 11 December 2014; Accepted 12 December 2014

Academic Editor: Mohammad Owais

Copyright (C) 2015 Gaetano Bertino et al. This is an open access article distributed under the Creative Commons Attribution License, which permits unrestricted use, distribution, and reproduction in any medium, provided the original work is properly cited.

Background. Hepatocellular carcinoma is a major health problem worldwide and the third most common cause of cancerrelated death. HCC treatment decisions are complex and dependent upon tumor staging. Several molecular targeted agents have been evaluated in clinical trials in advanced HCC. Despite of only modest objective response rates according to the Response Evaluation Criteria in Solid Tumors, several studies showed encouraging results in terms of prolongation of the time to progression, disease stabilization, and survival. Cellular immunotherapy would improve the immune state and has potential in enhancing the therapeutic outcome for HCC patients. Materials and Methods. A search of the literature was made using cancer literature, the PubMed, Scopus, and Web of Science (WOS) database for the following keywords: "hepatocellular carcinoma," "molecular hepatocarcinogenesis," "targeted therapy," "molecular immunological targets," "tumour-associated antigens," "Tregs," "MDSCs," "immunotherapy." Discussion and Conclusion. Treatment strategies combining blockade of immunoregulatory cell types such as Tregs and MDSCs and of inhibitory receptors, with vaccine-induced activation of TAA-specific T cells, may be necessary to achieve the most effective therapeutic antitumour activity in HCC. In the future, new therapeutic options will be represented by a blend of immunotherapy-like vaccines and T-cell modulators, supplemented by molecularly targeted inhibitors of tumor signaling pathways.

\section{Introduction}

Primary liver cancer is a major health problem worldwide. It is the fifth most common neoplasm in the world and the third most common cause of cancer-related death [1]. Over the past 15 years, the incidence of hepatocellular carcinoma (HCC) has more than doubled. Every year there are 500,000 new cases in the Asia-Pacific region, often due to chronic hepatitis B virus (HBV) infection [2]. More than $60 \%$ of a total number of HCC cases occur in China alone, and an estimated 360,000 patients residing in Far East countries, including China, Japan, Korea, and Taiwan, die from this disease each year. In Japan hepatitis $\mathrm{C}$ virus- ( $\mathrm{HCV}-)$ related HCC represents $70 \%$ of all cases [3]. In addition, in the USA and Europe, an increased incidence of HCV has led to an increased incidence of HCC [4]. A relevant risk factor for the high incidence of nonalcoholic fatty liver disease is obesity and diabetes, which can promote the development of liver cancer [5-7]. This involves a poor diagnosis and a low level of survival (5-year survival rate: less than 5\%) in patients 
with advanced HCC at diagnosis. Therefore, prevention and treatment of HCC are of great concern [8].

For a correct and effective HCC-surveillance in patients with cirrhosis, it is necessary to perform a liver ultrasound twice a year. Recently, the role of AFP serum levels has been discussed to be less useful than previously assumed [9-11]. Moreover, in addition to AFP, there are others that have been proposed as markers for early diagnosis of hepatocellular carcinoma. None of them is optimal; however, when used together, their sensitivity in detecting HCC is increased. Recent developments in gene-expressing microarrays and proteomics promise even more potential diagnostic options [12-20]. Immune-based therapy can represent an improvement in outcomes for patients with HCC, as many clinical trials demonstrate. Immunotherapy has been tested in HCC for many years. Most studies in the past have used either cytokine-based or antigen-based approaches [21, 22]. While most of these studies have proven to be safe and able to induce tumor-specific immune responses, most of them have failed to demonstrate clinical efficacy. We would like to summarize recent results from different immune-based approaches in HCC. These studies differ from previous immunotherapy studies in a number of respects: the targets; the quality of the immune response being activated; the approach taken to activate patients' immune system; and finally the more promising results seen.

\section{Molecular Immunological Targets}

The rationale for immunotherapy for HCC is based on the finding that patients with tumours, containing infiltrating, presumably tumour-specific effector $\mathrm{T}$ cells, had a reduced risk of tumour recurrence following liver transplantation [23]. Moreover, anti-CD3 and IL-2 stimulated autologous T lymphocytes infused in HCC patients significantly improved postsurgical recurrence-free survival [24]. These data imply a central role of $\mathrm{T}$ cells in modulating tumour progression and provide strong justification for T-cell immunotherapy.

At last, spontaneous antitumor responses have been detected in HCC patients. Activation of immune response and T-cell infiltration has been reported after percutaneous ethanol injection and radiofrequency ablation (RFA) $[25,26]$.

A prerequisite for the successful development of T-cellbased immunotherapeutic approaches is the identification and characterization of immune responses to tumour-associated antigens (TAAs). Seven HCC-specific TAAs that are targeted by $\mathrm{T}$ cells have been identified: alpha-fetoprotein (AFP), glypican-3 (GPC3), NY-ESO-1, SSX-2, melanoma antigen gene-A (MAGE-A), telomerase reverse transcriptase (TERT) [27-31], hepatocellular carcinoma-associated antigen-519/targeting protein for Xklp-2 (HCA519/TPX2) [32].

2.1. Alpha-Fetoprotein. AFP is expressed during foetal development but is transcriptionally repressed shortly after birth. However, it is reexpressed in the HCC patients. Since it is a self-protein, AFP-specific T-cell responses are suppressed and thus difficult to activate. Nevertheless, AFP is currently the best studied and most promising target antigen for HCC immunotherapy. The Butterfield LH and colleagues identified an HLA-A2-restricted AFP-specific CD8 T-cell epitope and demonstrated its ability to generate CD8+ T-cell responses both in human lymphocyte cultures and in HLA-A2 transgenic mice. The authors further identified four dominant and 10 subdominant AFP-specific epitopes, all of which induced low to moderate CD8+ T-cell responses in PBMCs of HCC patients [33]. Thimme et al. (2008) have compared AFP-specific CD8+ T-cell responses in PBMCs with those in tumour-infiltrating lymphocytes (TILs) and surrounding nontumour liver tissue by stimulating lymphocytes with 18 mer overlapping peptides spanning the entire AFP protein. The authors found T-cell responses against several previously unidentified epitopes without a clear immunodominance, indicating that more AFP-specific epitopes exist and need to be identified [27]. These results also indicate that CD8+ $\mathrm{T}$ cells specific for the self-antigen AFP are present in the normal T-cell repertoire and are not centrally or peripherally deleted.

2.2. Glypican-3. GPC3 is a foetal oncoprotein. It belongs to a family of heparan sulphate proteoglycans that can bind growth factors (Wnt, FGF1/2, and VEGF) and thereby promote tumour growth. GPC3 was found to be overexpressed in HCC and is considered a promising target antigen. Two HLAA2-and HLA-24-restricted CD8 T-cell epitopes of GPC3 have been mapped in mice and shown to elicit T-cell responses in HCC patients after long-term in vitro stimulation of PBMCs [34].

2.3. NY-ESO-1. Expression of NY-ESO-1 is limited to testis in healthy subjects; however, it is often expressed de novo in HCC and other cancers such as melanoma, ovarian, and breast cancer. NY-ESO-1 expression is associated with worse HCC outcome following surgery, and the mechanism for this finding may be that NY-ESO-1 increases tumor cell migration [35]. NY-ESO-1 is one of the most immunogenic TAAs known to date, with several identified epitopes currently being tested as potential vaccine candidates for cancers other than HCC. By using different approaches, three groups reported NYESO-1-specific CD8+ T-cell responses in the peripheral blood of HCC patients to an epitope that had already been identified in melanoma patients $[29,36]$. None of the observed T-cell responses were, however, detectable ex vivo and required several weeks of in vitro lymphocyte expansion, suggesting a relatively low frequency of these cells in the circulation.

2.4. SSX-2. SSX-2 is a cancer-testis antigen that has been demonstrated to be overexpressed in HCC patients. By using a CD8 T-cell epitope that had been previously identified in melanoma patients, two groups detected CD8+ T-cell responses in a small fraction of HCC patients after in vitro expansion of PBMCs and TILs [37, 38].

2.5. Melanoma Antigen Gene-A. Antigens of the MAGE-A family were originally characterized in melanoma, but they are also widely expressed in other tumours such as breast carcinoma, glioma, colorectal carcinoma, and HCC. Zerbini et al. [39] demonstrated in vitro induction of CD8+ T-cell 
responses against previously identified MAGE-A1 and MAGE-A3 epitopes in tumour infiltrating but not peripheral blood lymphocytes derived from HCC patients. CD8+ T-cell responses against MAGE-A10 epitope have also been described by Bricard et al. [37]. Therefore, MAGE-A3-specific TCRs are identified as disease markers and made ready for immune therapy.

2.6. Telomerase Reverse Transcriptase. TERT is a catalytic enzyme required for telomere elongation; its expression correlates with telomerase activity. Tumours must retain the length of their telomeres and telomerase helps them to do so by maintaining telomeric ends of linear chromosomes and protecting them from degradation. It is, therefore, not surprising that telomerase has been reported to be activated in up to $85 \%$ of all human cancers, including HCC, whereas TERT has been proposed as a universal TAA for cancer immunotherapy [40]. Mizukoshi and colleagues reported ex vivo measurable weak CD8+ T-cell responses to six TRET epitopes in the peripheral blood of HCC patients [31].

2.7. Hepatocellular Carcinoma-Associated Antigen-519/Targeting Protein for Xklp-2. One HCC-associated antigen previously defined by a humoral response is HCA519.14. HCA519, also known as targeting protein for Xklp-2 (TPX2), is a microtubule associated protein needed for HCC cell division. HCA519/TPX2 associates with microtubules via kinetochores and facilitates aurora kinase A binding to the microtubules [41]. Enhanced TPX2 production has also been associated with pancreatic and lung cancers $[42,43]$. Studies by Tanaka et al. [44] and Yang et al. [45] show upregulation of TPX2 within HCC by microarray analysis. Satow et al. demonstrated higher expression of HCA519/TPX2 in HCC tumor tissue compared to adjacent nontumor tissue [46]. Small interfering- (siRNA-) mediated knockdown of this gene inhibited the proliferation of HCC cells and prevented the growth of HCC xenografts injected into immunodeficient mice. So this antigen might be at a key spot in the HCC replication cycle, where immunotherapy could have an immediate effect. Even if only the highly expressing HCA519/TPX2 + HCC cells are killed by activated CTLs, then the HCC tumor cell population as a whole might be sufficiently reduced, thereby hindering the in vivo tumor growth and in turn providing the patient with a longer survival. Thus, HCA519/TPX2 may be developed into future immunotherapy for HCC, which targets a key protein that these cancer cells need for their proliferation. This new antigen for HCC might be beneficial for T-cell immunotherapy HCC [40].

\section{Alteration of Different Pattern of Immune System}

In patients with HCC, there is an alteration of different pattern of immune system.

Several mechanisms could contribute to the weak and often inefficient TAA-specific CD8+ T-cell responses in HCC patients.

(i) The regulatory $\mathrm{T}$ cells (Tregs). Regulatory $\mathrm{T}$ lymphocytes are able to inhibit the T-helper response and a cytokine profile secretion which is distinct from those of Th1 and Th2 cells. Even if many Tregs subpopulations have been described, $\mathrm{CD} 4+\mathrm{CD} 25+$ and Type 1 regulatory $\mathrm{T}$ cells ( $\mathrm{Tr} 1)$ are the best characterized. CD4+CD25+ are induced in the thymus and interleukin 2 appears to be fundamental for their survival, expansion, and suppressive function [47]. Once activated, they can suppress CD4+ and CD8+ effector T-cell proliferation and cytokine secretion and inhibit B lymphocytes proliferation [48-50]. Moreover, their depletion is correlated to a more efficient antitumoral immune response [51, 52]. Type 1 regulatory $\mathrm{T}$ cells, instead, produce a large amount of interleukin 10 and transforming grow factor beta (TGF-b) that inhibit type 1 and type 2 helper response. Unlike CD4+CD25+ Tregs, their proliferative factor is interleukin 15, not interleukin 2. Type 1 regulatory $T$ cells could suppress the effector function of CD8+ $\mathrm{T}$ cells [53]. As a consequence of the immunosuppression phenomena related to these particular Treg subpopulations, these cells can be responsible in part for the development of virus-induced HCC [54-57].

(ii) Myeloid-Derived Suppressor Cells (MDSCs). Myeloidderived suppressor cells comprise a mixture of monocytes/macrophages, granulocytes, and dendritic cells $(D C s)$ at different stages of differentiation. In patients with HCC, blood MDSCs have recently been shown to induce Foxp3 and IL-10 expression in CD4+ T cells via arginase activity $[58,59]$.

MDSCs are immature/progenitor myeloid cells with immunosuppressive and proangiogenic activity via a variety of direct and indirect mechanism that targets DCs, T cells, and macrophages. They maintain an immature phenotype when exposed to proinflammatory signals and contribute to a tumor-promoting type 2 phenotype by their production of interleukin 10 and their blocking of macrophage production of interleukin 12 [60]. MDSC accumulation is found not only within the tumors but also in blood circulation, spleen, bone marrow, and liver [61]. The MDSCs inhibit the function of effector T cells and decrease NK cells cytotoxicity, cytokine production, and maturation of DC [62]. Hoechst et al. suggested that MDSCs can inhibit and regulate NK cells as effectors of the innate immune system contributing further to immune suppressor mechanisms in patients with HCC [63]. The frequency of MDSCs has been shown to correlate with recurrence-free survival of HCC patients who have undergone RFA [64-66]. It has also been suggested that MDSCs interact with Kuppfer cells to induce PDL1 expression, which in turn inhibits antigen presentation. They facilitate tumor progression by their cross-talk with immune cells, resulting in a decrease in production of interleukin 12 and T-cell stimulatory activity. The MDSC-induced downregulation of macrophage production of interleukin 12 is dependent on MDSC synthesis of interleukin 10 [60]. MDSCs may also help expand Treg population. Depletion of Tregs or MDSCs could prompt spontaneous immune responses against AFP, suggesting the potential of immune reactivation [67]. Recently, Han et al. [68] have identified new 


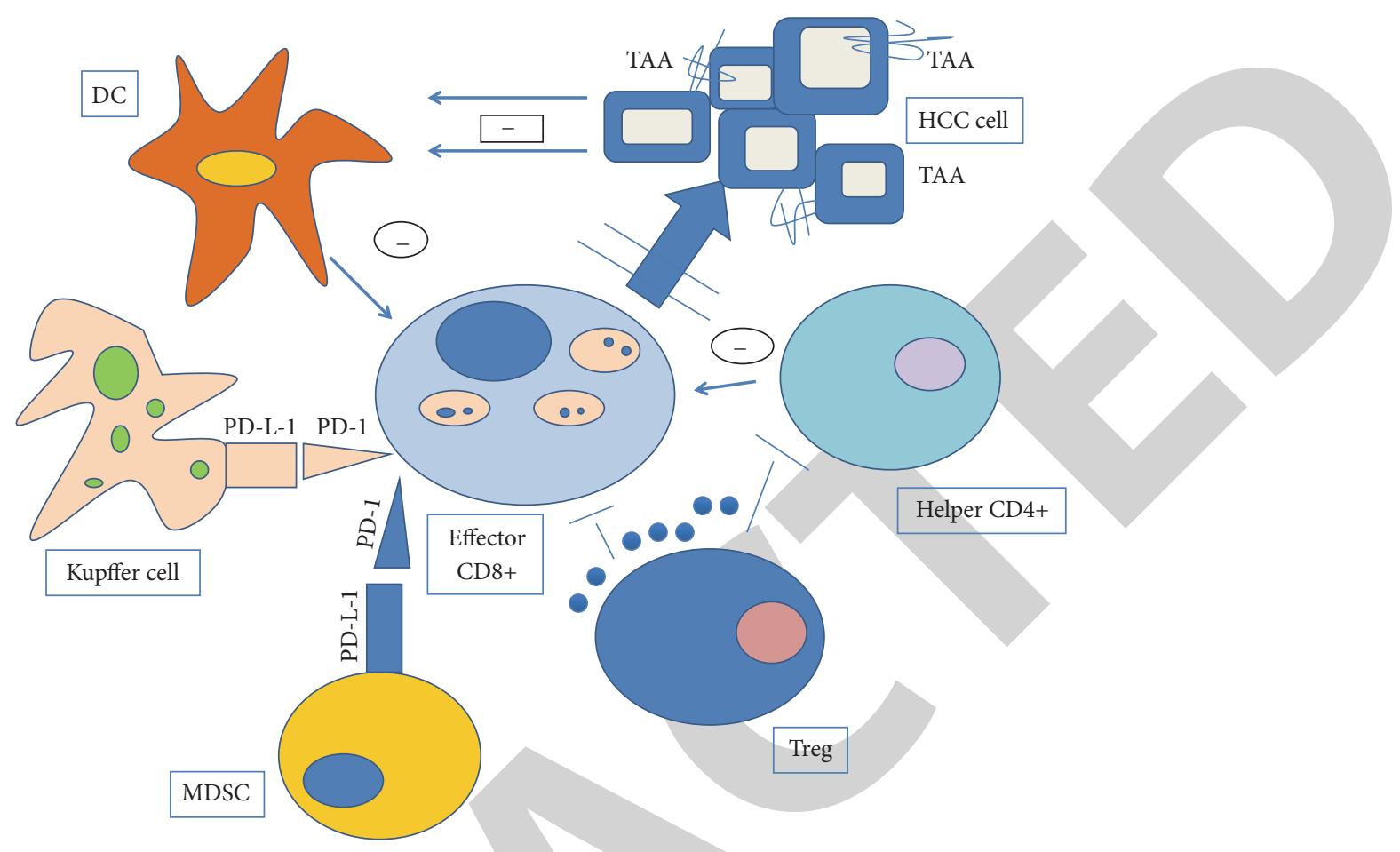

FIGURE 1: Mechanisms responsible for inefficient T-cell responses in HCC. Failure of TAA processing and presentation; suppression of CD4+ and CD8+ cells by Treg; insufficient levels of CD4 help; negative regulation by PD-1/PDL1 pathway.

subset of immune suppressive cells in HCC patients called regulatory DCs. These regulatory DCs can suppress T-cell activation through interleukin (IL-) 10 and indoleamine 2,3dioxygenase (IDO) production $[68,69]$. There is currently no specific drug or antibody available that selectively targets MDSCs. Since inhibition of arginase activity can cause side effects, due to the critical role of this enzyme in the urea cycle, it will be important to identify additional specific markers to target these cells [70].

(i) Impairment of TAA processing and presentation. Several studies have shown that expression of HLA class I molecules and B7 costimulatory molecules is downregulated in HCC tissue and HCC cell lines. Such downregulation is likely to lead to impaired processing of TAAs. Moreover, it has been shown that circulating myeloid DCs in HCC patients were decreased in numbers and had reduced cytokine production, raising the possibility that TAA presentation by DCs may also be impaired [71-73].

(ii) Inhibitory receptors. Many human cancers express PD-L1, the ligand for the inhibitory receptor programmed cell death-1 (PD-1). Tumour-associated PD-L1 has been shown to induce apoptosis of effector $\mathrm{T}$ cells and is thought to contribute to immune evasion by cancers. In HCC, tumour infiltrating CD $8+\mathrm{T}$ cells are characterized by an increase in PD-1 expression. Intratumour Kupffer cells have been shown to upregulate $\mathrm{PD}-\mathrm{L} 1$ and decrease the effector function of PD-1-expressing CD8+ T cells in HCC patients. It should be noted that cells with a MDSC phenotype also upregulated PD-L1 in these studies and exerted a similar inhibitory effect on T-cell activation, raising the possibility of MDSC-mediated T-cell suppression. These data suggest that the inhibition of PD-1 may be a potential strategy in the boosting of HCC-specific immunity [74-77].

(iii) Lack of CD4+ T-cell responses. It is known that a lack of CD4+ T-cell help may lead to CD8+ T-cell exhaustion. In fact, in HCC, AFP-specific CD4+ Tcell responses were only present in patients with early stage disease and became exhausted as the disease progressed. Thus, for the activation of fully functional cytotoxic $\mathrm{T}$ lymphocytes, it will be important to identify TAA-derived CD4 T helper cell epitopes and include them in a vaccine along with CD8 T-cell epitopes.

In conclusion, multiple mechanisms may limit the TAA-specific CD8+ T-cell responses in HCC: failure of TAA processing and presentation; insufficient levels of CD4 help; suppression of both CD4+ and CD8+ T cells by Tregs; negative regulation by $\mathrm{PD}-1 / \mathrm{PD}-\mathrm{L} 1$ pathway (Figure 1).

\section{Immunosuppression Induced by Chronic HBV and HCV Infection}

In HCC, there is chronic hepatitis B virus and hepatitis C virus-mediated immunosuppression. Chronic $\mathrm{HBV}$ or $\mathrm{HCV}$ infections is well known to induce a chronic proinflammatory hepatic and systemic state associated with immunosuppressive and immunomodulatory effects [57, 78-85]. 
Dysfunctional T-cell responses to both virus-specific and unrelated antigens, characterized by impaired proliferation and IL-2 production, are observed in chronic hepatitis B virus-infected patients. Chronic infection with hepatitis $\mathrm{C}$ virus negatively regulates both the innate and adaptive arms of the immune system. There are many mechanisms of HCC immune escape; the peripheral blood of HCC patients shows impaired IL-12 production and reduced allostimulatory activity [86]. In HCC numerous regulatory mechanisms involving nearly all cellular subsets of the immune system contribute to tumor development and progression. These suppressor mechanisms range from $\mathrm{CD} 4+$ regulatory $\mathrm{T}$ cells, functionally impaired dendritic cells, neutrophils, and monocytes to myeloid derived suppressor cells. An impairment of natural killer cell production and activity has also been described in HCC patients [87-89]. In vitro as well as in vivo studies have demonstrated that abrogation of the suppressor cells enhances or unmasks tumor specific antitumor immune responses [90].

\section{Current and Future Immunotherapy of HCC}

Cellular immunotherapy would improve the immune state and has potential in enhancing the therapeutic outcome for HCC patients. Current attempts at harnessing the immune system to eliminate tumors have been focusing on vaccination, such as a dendritic cell (DC) vaccine, to increase the frequency of tumor specific cytotoxic $\mathrm{T}$ lymphocytes and adoptive transfer of effector $\mathrm{T}$ cells to promote tumor regression [91]. However, despite considerable success in preclinical studies, the outcome of immunotherapy is often disappointing when translated to clinical trials, which is at least in part due to the complexity of the immune escape mechanism of tumor cells.

Immune-based therapy could improve outcome for patients with HCC and some studies have been developed to obtain evidence to support this hypothesis, but not in controlled trials.

(i) In a historical study, 150 patients were randomized to receive either IL-2 and anti-CD3-activated PBMC, or observation after curative resection [24]. The results were encouraging, both with respect to time to relapse and disease-free survival $(P=0.09)$. A trial testing the administration of APC in HCC patients who received pulsed DC with autologous tumor lysate [92] showed an increase of 1-year survival (63 versus $10 \%$; $P=0.038$ ). DCs could be used as a potential cellular adjuvant for the production of specific tumor vaccines.

(ii) Recently, El Ansary and colleagues's study [89] evaluated the safety and efficacy of the autologous pulsed DC vaccine in advanced HCC patients in comparison with supportive treatment. Thirty patients with advanced HCC who were not suitable for radical or loco regional therapies were enrolled. Patients were divided into two groups, group I, consisting of 15 patients, received vaccination with mature autologous DCs pulsed ex vivo with a liver tumor cell line lysate.
Group II (control group; $n=15$ ) received supportive treatment. To generate DCs, 100 and $4 \mathrm{~mL}$ of venous blood were obtained from each patient. DCs were identified by CD80, CD83, CD86, and HLA-DR expressions using flow cytometry. Follow-up at 3 and 6 months after injection by clinical, radiological, and laboratory assessment was carried out. Improvements in OS were observed. Partial radiological response was obtained in two patients (13.3\%), stable course was obtained in nine patients $(60 \%)$, and four patients (26.7\%) showed progressive disease (died at 4 months after injection). Both CD8+ T cells and serum IFN$\mathrm{g}$ were elevated after DC injection. The authors conclude that autologous DC vaccination in advanced HCC patients is safe and well tolerated.

Ex vivo treatment with cytotoxic $\mathrm{T}$ lymphocyte associated antigen-4 (CTLA-4) blocking antibodies of T-cell CD8+, isolated from patients affected by HCC, showed an expanded antigen-specific T-cell repertoire, alluding that ipilimumab and tremelimumab may possess a therapeutic potential in treating hepatocarcinoma [93].

(i) The US FDA approved ipilimumab for the treatment of melanoma in 2011 based on randomized phase III trial showing improved overall survival $[94,95]$.

(ii) Tremelimumab is a monoclonal antibody that blocks cytotoxic T lymphocyte-associated antigen 4 (CTLA4), an inhibitory coreceptor that interferes with T-cell activation and proliferation. Tremelimumab showed early evidence of antitumor activity in a single arm phase II trial in malignant mesothelioma [96]. A phase II trial of tremelimumab in HCC patients has recently been reported (NCT01008358) [97]. The study enrolled 21 patients with chronic hepatitis $\mathrm{C}$ with Child-Pugh A or B cirrhosis and advanced HCC not amenable to percutaneous ablation or transarterial embolization. Partial responses were seen in $17.6 \%$ of the cases, and $45 \%$ of the patients had stable disease for more than 6 months. Interestingly, the patients with stable levels of interferon- (IFN-) $\gamma$ during the treatment showed better treatment response compared to those who showed a decrease, suggestive of more active antitumor immunity. A phase I clinical trial of tremelimumab with radiofrequency ablation or transarterial therapy is ongoing (NCT01853618). These data suggest that the efficacy of CTLA- 4 blockade may correlate with the immunogenicity of the tumor. Secondly, the immunotherapy may be more effective against smaller tumors [98].

(iii) PD-1 blockade: a therapeutic advantage, regarding refractory solid tumors, can be obtained by an antibody-mediated block of PD-1; meanwhile the inhibition of Tim-3 signaling has been demonstrated to restore antitumor T-cell action in preclinical models [99]. Anti-PD-1 antibodies and 3 anti-PD-L1 antibodies are currently under development, emphasizing the growing interest in this immune checkpoint pathway as a target for cancer therapy. 
(iv) Lambrolizumab induced tumor regression in advanced melanoma patients and showed a favorable safety profile [100]. Lambrolizumab was effective even in patients who failed ipilimumab treatment, which suggests a differential mechanism of action for PD1 inhibition versus CTLA-4 blockade. Indeed, combination of ipilimumab with nivolumab achieved objective response in $40 \%$ of the patients [101].

(v) CT-011 and MPDL3280A/RG7446 were tested in phase I trials and showed with favorable safety profiles [102].

(vi) MEDI4376 targets PD-L1, and phase I trial is ongoing.

(vii) AMP-224 is a recombinant B7-DC-Fc fusion protein, and a phase I trial of this agent is also underway. In HCC, a phase I/II trial of CT-011 in advanced HCC was initiated but stopped due to slow accrual.

(viii) A phase I trial of nivolumab is currently ongoing for patients with advanced HCC (NCT01658878). A phase I dose escalation study to investigate the safety, immunoregulatory activity, pharmacokinetics, and preliminary antitumor activity of anti-programmeddeath-1 (PD-1) antibody (BMS-936558) in advanced hepatocellular carcinoma in subjects with or without chronic viral hepatitis. This study plans to enroll three cohorts of patients stratified by viral etiology (HBV, $\mathrm{HCV}$ ) and no viral infection, with a target enrollment of 72 patients.

At present, several clinical trials, that are studying the quoted molecules, are ongoing in order to get evidences for a possible approval for the combination therapy to treat HCC. At the moment sorafenib is the only drug approved by FDA with a specific indication for HCC (Table 1). Another approach has been described to overcome cancermediated immunosoppression, involving the reactivation of hyporesponsive tumor-specific $\mathrm{T}$ cells by supplying $\mathrm{T}$-cell growth factors (IL-15 and IL-7) or costimulatory agonists (anti-4-1BB and anti-OX40) [103]. Other treatment options regarding tumor homing and penetration of T-effector cells are being evaluated because of the correlation between $\mathrm{T}$ cell infiltration of hepatocarcinoma lesions and OS. Strategies are divided into two big groups: restoring the tumor vascularity and upregulation of chemokines and molecules of adhesion. Monoclonal antibodies against VEGF and its receptors, such as sorafenib or bevacizumab, appear to have a restricted therapeutic effect in clinical trials [104, 105]. In fact a hallmark of new vessel formation in HCC is their structural and functional abnormality; this leads to an abnormal tumor microenvironment characterized by low oxygen tension and low therapeutic agent levels [106]. Preclinical data sustain the idea that angiogenesis and tumor vascularity still represent a potential target that, through the generation of long-lived antivascular T-cell responses via VEGFR2 vaccine, can be suppressed via a T-cell dependent process [89]. Proinflammatory chemokines demonstrated their importance in HCC-specific T-cell immunity, such as IFN-ginducible chemokines CXCL9/Mig and CXCL10/IP-10, high levels of which correlated with the presence of CD8+ T cell in hepatocarcinoma. It is still unknown if this pattern of chemokine expression is correlated with a positive prognosis, as has been seen in patients with cervical/uterine tumors. Agents that can induce the expression of chemokines and adhesion molecules by vascular activation represent another promising approach [107].

\section{Discussion and Conclusion}

HCC treatment decisions are complex and dependent upon tumor staging. In patients with unresectable disease and tumor staging that falls within criteria, liver transplantation can be curative in a great majority of patients. Unfortunately, most patients will not be candidates for either surgery or transplant [108-110].

Cytotoxic chemotherapy and hormonal agents have been tested in HCC with marginal efficacy to date. Recent insights into the molecular pathogenesis of HCC have identified several aberrant signaling pathways that have served as targets for novel therapeutic agents. Several pathways are now implicated in hepatocarcinogenesis and agents that target these pathways continue to be developed.

The knowledge of molecular hepatocarcinogenesis broadened the horizon for patients with advanced HCC. During the last years, several molecular targeted agents have been evaluated in clinical trials in advanced HCC. Despite of only modest objective response rates according to the Response Evaluation Criteria in Solid Tumors (RECIST) [111], several studies showed encouraging results in terms of prolongation of the time to progression (TTP), disease stabilization (DS), and survival.

Cellular immunotherapy would improve the immune state and has potential in enhancing the therapeutic outcome for HCC patients. Immune-based therapy could improve outcome for patients with HCC, but we will need controlled studies demonstrating the validity of this hypothesis.

A prerequisite for the successful development of T-cellbased immunotherapeutic approaches is the identification and characterization of immune responses to tumour-associated antigens (TAAs).

In patients with HCC, there is an alteration of different pattern of immune system; therefore it is necessary to study the different mechanisms that could weaken and make often inefficient TAA-specific CD8+ T-cell responses in patients affected by HCC.

Treatment strategies combining blockade of immunoregulatory cell types such as Tregs and MDSCs and of inhibitory receptors, with vaccine-induced activation of TAA-specific T cells, may be necessary to achieve the most effective therapeutic anti-tumour activity in HCC. Also, a combination with conventional HCC treatments, such as transarterial embolization or local tumour ablation may increase HCC immunogenicity and unmask TAA-specific T-cell responses [112].

Data from clinical trials completed permit to imagine that immunotherapy with immune blockers checkpoint antibodies may represent an effective strategy in the treatment of advanced HCC. PD-1/PD-L1 plays a pivotal role in tumor evasion and blocking interaction with anti-PD-L1 antibody would revitalize CD8+ T cells to give them a second chance 


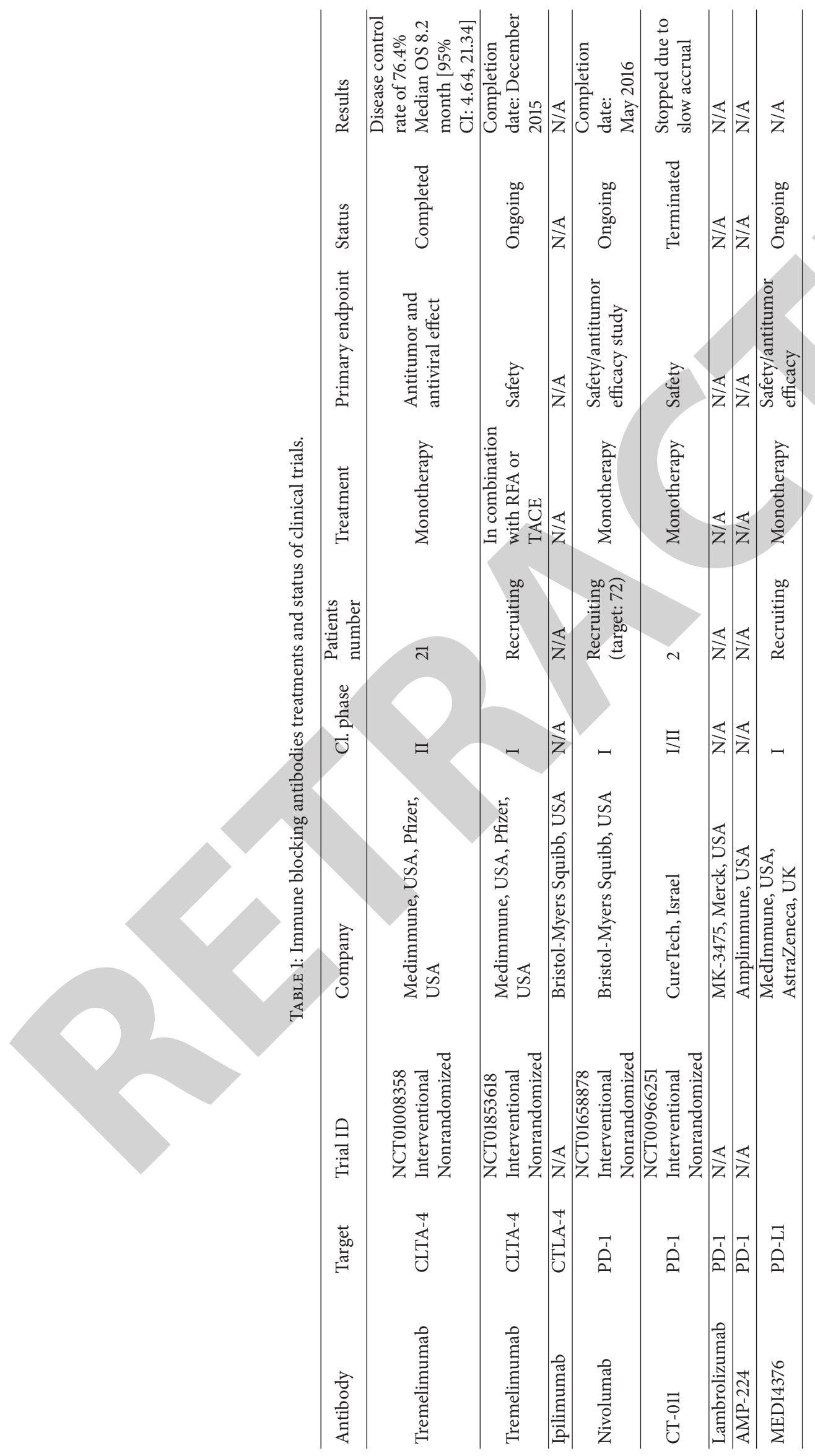


to clear the tumor. Unfortunately, there are no solid data on the use of PD-blockade alone. Therefore, it is likely that the more realistic approach would be made up of the combined use of CTLA-4 blocking antibodies with TACE and RFA or with anti-PD-1 antibodies.

As DCs are specialized for antigen presentation and their immunogenicity leads to the induction of antigen-specific immune responses, various immunotherapeutic approaches have been designed for using DCs to present tumor-associated antigens to T lymphocytes. As part of a strategy proposed ex vivo generated DCs might be loaded with antigens and reinfused to the patients and/or they can be used for the ex vivo expansion of antitumor lymphocytes. The DCs loaded ex vivo with RNA can be safely administered which proves to be an asset for producing antigen-specific immune responses. DCs could be used as potential cellular adjuvant for the production of specific tumor vaccines. Autologous DC vaccination in advanced HCC patients is safe and well tolerated but, for the complex pathogenesis of HCC, does not seem to be able by itself to restore an effective anti-tumor response.

In the near future we need to consider some important aspects: the therapeutic strategy will be most likely to succeed if the development will be guided by biology-driven biomarkers of response to longer available agents; the therapy with immune checkpoint blockers is most likely to succeed in combination with other surgical, cytotoxic, or immune targeted therapies; integration of immune checkpoint blockers in combination with other agents will have to address the safety concerns specific to this population of patients, such as hepatotoxicity. Expand knowledge on these issues could contribute to the success of new immunotherapies.

Molecular alterations may differ depending on the underlying risk factors and etiologies, potentially influencing patient responses to therapy. Thus, it will be necessary to classify HCCs into subgroups according to their genomic and proteomic profiling. The identification of the key molecules/receptors/signaling pathways and the assessment of their relevance as potential targets will be the main future challenge.

In this context the study of small molecules interfering RNA such as siRNA and their relations with membrane glycoproteins such as integrins is very intriguing.

Integrins play an important role during development, regulating cell differentiation, proliferation, and survival. It was demonstrated that knockdown of integrin subunits slows the progression of hepatocellular carcinoma. Delivery using nanoparticles of short interfering RNA directed $\beta 1$ and $\alpha \mathrm{v}$ integrin subunits downregulated all integrin receptors on hepatocytes. Short term integrin knockdown causes no apparent structural or functional disruption of normal liver tissue. Alteration of the morphology of the liver accumulates on sustained integrin downregulation. The integrin knockdown leads to significant delays in HCC progression, reducing the proliferation and increased tumor cell death. This delay tumor is accompanied by reduced MET oncogene activation and expression of its mature form on the cell surface. These data suggest that the proliferating cells transformed by HCC are more sensitive to integrin knockdown of normal quiescent hepatocytes, highlighting the potential of siRNA-mediated inhibition of integrins as an anticancer therapeutic approach.

siRNA, also known as short interfering RNA or silencing RNA, is a class of double-stranded RNA molecules, 2025 base pairs in length. siRNA plays many roles but is most notable in the path RNA interference (RNAi), which interferes with the expression of specific genes with complementary nucleotide sequences. siRNA functions by causing mRNA to be broken down after transcription, resulting in no translation. siRNA also acts in complexity pathways. The RNAi related to these pathways is only now being elucidated.

It is important to note that normal hepatocytes can, at least in part, adapt to the decrease in the level of integrin signaling, in contrast to the tumor cells and isolated cells in vitro and proliferating hepatocytes in regenerating liver. Inhibition of integrin receptors, including Itgav subunit, is often referred to as cancer therapy effective by acting directly on tumor cells and as antiangiogenic factor. These results, together with others, indicate a high therapeutic potential of anti-ItgblsiRNA. In light of the results that inhibition of signaling Itgbl can increase the sensitivity of the tumor to radiation and chemotherapy, Itgbl specific siRNA can be proposed as part of a combination therapy [113].

In addition the macrophage migration inhibitory factor (MIF), a proinflammatory and immunoregulatory chemokine, plays important roles in cancer-related biological processes. However, few studies have focused on the clinical relevance of MIF and cyclin D1 expression in hepatocellular carcinoma cells (HCCs).

siRNA-mediated knockdown of MIF suppressed cyclin D1 expression and hepatocellular carcinoma cell proliferation. MIF siRNA reduces proliferation and increases apoptosis in HCC cells. MIF knockdown inhibits the expression of growth-related proteins and induces the expression of apoptosis-related proteins, supporting a role for MIF as a novel therapeutic target for HCC [114].

Therefore, in the future, new therapeutic options will be represented by a blend of immunotherapy-like vaccines and T-cell modulators, supplemented by molecularly targeted inhibitors of tumor signaling pathways [115-119].

\section{Conflict of Interests}

The authors declare that there is no conflict of interests regarding the publication of this paper.

\section{References}

[1] K. J. Lafaro, A. N. Demirjian, and T. M. Pawlik, "Epidemiology of hepatocellular carcinoma," Surgical Oncology Clinics of North America, vol. 24, no. 1, pp. 1-17, 2014.

[2] C.-L. Lin and J.-H. Kao, "Risk stratification for hepatitis B virus related hepatocellular carcinoma," Journal of Gastroenterology and Hepatology, vol. 28, no. 1, pp. 10-17, 2013.

[3] T. Umemura, T. Ichijo, K. Yoshizawa, E. Tanaka, and K. Kiyosawa, "Epidemiology of hepatocellular carcinoma in Japan," Journal of Gastroenterology, vol. 44, supplement, no. 19, pp. 102107, 2009.

[4] J. A. Davila, J. R. Kramer, Z. Duan et al., "Referral and receipt of treatment for hepatocellular carcinoma in United States 
veterans: effect of patient and nonpatient factors," Hepatology, vol. 57, no. 5, pp. 1858-1868, 2013.

[5] G. Baffy, E. M. Brunt, and S. H. Caldwell, "Hepatocellular carcinoma in non-alcoholic fatty liver disease: an emerging menace," Journal of Hepatology, vol. 56, no. 6, pp. 1384-1391, 2012.

[6] G. Bertino, A. M. Ardiri, F. T. Alí et al., "Obesity and related diseases: an epidemiologic study in eastern Sicily," Minerva Gastroenterologica e Dietologica, vol. 52, no. 4, pp. 379-385, 2006.

[7] M. Malaguarnera, M. Vacante, C. Russo et al., "Rosuvastatin reduces nonalcoholic fatty liver disease in patients with chronic hepatitis $\mathrm{C}$ treated with $\alpha$-interferon and ribavirin: rosuvastatin reduces NAFLD in HCV patients," Hepatitis Monthly, vol. 11, no. 2, pp. 92-98, 2011.

[8] J. M. Llovet, A. Burroughs, and J. Bruix, "Hepatocellular carcinoma," The Lancet, vol. 362, no. 9399, pp. 1907-1917, 2003.

[9] A. Toro, A. Ardiri, M. Mannino et al., "Effect of pre- and posttreatment $\alpha$-fetoprotein levels and tumor size on survival of patients with hepatocellular carcinoma treated by resection, transarterial chemoembolization or radiofrequency ablation: a retrospective study," BMC Surgery, vol. 14, no. 1, p. 40, 2014.

[10] I. Di Carlo, M. Mannino, A. Toro et al., "Persistent increase in alpha-fetoprotein level in a patient without underlying liver disease who underwent curative resection of hepatocellular carcinoma. A case report and review of the literature," World Journal of Surgical Oncology, vol. 10, article 79, 2012.

[11] G. Bertino, A. M. Ardiri, M. M. Santonocito, and P. M. Boemi, "Some patients with HCC haven't abnornormal des-gammacarboxy prothrombin and alpha-fetoprotein levels," Panminerva Medica, vol. 51, no. 2, pp. 133-134, 2009.

[12] G. Bertino, A. Ardiri, M. Malaguarnera, G. Malaguarnera, N. Bertino, and G. S. Calvagno, "Hepatocellualar carcinoma serum markers," Seminars in Oncology, vol. 39, no. 4, pp. 410-433, 2012.

[13] G. Bertino, S. Neri, C. M. Bruno et al., "Diagnostic and prognostic value of alpha-fetoprotein, des- $\gamma$-carboxy prothrombin and squamous cell carcinoma antigen immunoglobulin $\mathrm{M}$ complexes in hepatocellular carcinoma," Minerva Medica, vol. 102, no. 5, pp. 363-371, 2011.

[14] G. Bertino, A. M. Ardiri, G. S. Calvagno, N. Bertino, and P. M. Boemi, "Prognostic and diagnostic value of des- $\gamma$-carboxy prothrombin in liver cancer," Drug News and Perspectives, vol. 23, no. 8, pp. 498-508, 2010.

[15] G. Bertino, A. M. Ardiri, P. M. Boemi et al., "A study about mechanisms of des-gamma-carboxy prothrombin's production in hepatocellular carcinoma," Panminerva Medica, vol. 50, no. 3, pp. 221-226, 2008.

[16] A. Biondi, G. Malaguarnera, M. Vacante et al., "Elevated serum levels of Chromogranin A in hepatocellular carcinoma," BMC Surgery, vol. 12, article 7, supplement 1, 2012.

[17] G. Malaguarnera, I. Paladina, M. Giordano, M. Malaguarnera, G. Bertino, and M. Berretta, "Serum markers of intrahepatic cholangiocarcinoma," Disease Markers, vol. 34, no. 4, pp. 219228,2013

[18] G. Bertino, A. Ardiri, S. Demma et al., "Rare benign tumors of the liver: still rare?" Journal of Gastrointestinal Cancer, vol. 45, no. 2, pp. 202-217, 2014.

[19] A. Toro, A. E. Mahfouz, A. Ardiri et al., "What is changing in indications and treatment of hepatic hemangiomas. A review," Annals of Hepatology, vol. 13, no. 4, pp. 327-339, 2014.

[20] G. Bertino, A. M. Ardiri, G. S. Calvagno et al., "Carbohydrate 19.9 antigen serum levels in liver disease," BioMed Research International, vol. 2013, Article ID 531640, 6 pages, 2013.
[21] T. F. Greten, M. P. Manns, and F. Korangy, "Immunotherapy of hepatocellular carcinoma," Journal of Hepatology, vol. 45, no. 6 , pp. 868-878, 2006.

[22] T. F. Greten, M. P. Manns, and F. Korangy, "Immunotherapy of HCC," Reviews on Recent Clinical Trials, vol. 3, no. 1, pp. 31-39, 2008.

[23] E. Unitt, A. Marshall, W. Gelson et al., "Tumour lymphocytic infiltrate and recurrence of hepatocellular carcinoma following liver transplantation," Journal of Hepatology, vol. 45, no. 2, pp. 246-253, 2006.

[24] T. Takayama, T. Sekine, M. Makuuchi et al., "Adoptive immunotherapy to lower postsurgical recurrence rates of hepatocellular carcinoma: a randomised trial," The Lancet, vol. 356, no. 9232, pp. 802-807, 2000.

[25] K. Ohnishi, H. Yoshioka, S. Ito, and K. Fujiwara, "Prospective randomized controlled trial comparing percutaneous acetic acid injection and percutaneous ethanol injection for small hepatocellular carcinoma," Hepatology, vol. 27, no. 1, pp. 67-72, 1998.

[26] S. Shiina, K. Tagawa, T. Unuma et al., "Percutaneous ethanol injection therapy for hepatocellular carcinoma: a histopathologic study," Cancer, vol. 68, no. 7, pp. 1524-1530, 1991.

[27] R. Thimme, M. Neagu, T. Boettler et al., "Comprehensive analysis of the $\alpha$-fetoprotein-specific $\mathrm{CD} 8^{+} \mathrm{T}$ cell responses in patients with hepatocellular carcinoma," Hepatology, vol. 48, no. 6, pp. 1821-1833, 2008.

[28] H. Komori, T. Nakatsura, S. Senju et al., "Identification of HLA-A2- or HLA-A24-restricted CTL epitopes possibly useful for glypican-3-specific immunotherapy of hepatocellular carcinoma," Clinical Cancer Research, vol. 12, no. 9, pp. 2689-2697, 2006.

[29] X.-Y. Shang, H.-S. Chen, H.-G. Zhang et al., "The spontaneous $\mathrm{CD}^{+}$T-cell response to HLA-A2-restricted NY-ESO-1b peptide in hepatocellular carcinoma patients," Clinical Cancer Research, vol. 10, no. 20, pp. 6946-6955, 2004.

[30] G. Bricard, H. Bouzourene, O. Martinet et al., "Naturally acquired MAGE-A10- and SSX-2-specific $\mathrm{CD} 8^{+} \mathrm{T}$ cell responses in patients with hepatocellular carcinoma," The Journal of Immunology, vol. 174, no. 3, pp. 1709-1716, 2005.

[31] E. Mizukoshi, Y. Nakamoto, Y. Marukawa et al., "Cytotoxic T cell responses to human telomerase reverse transcriptase in patients with hepatocellular carcinoma," Hepatology, vol. 43, no. 6, pp. 1284-1294, 2006.

[32] A. M. Aref, N. T. Hoa, L. Ge et al., "HCA519/TPX2: a potential T-cell tumor-associated antigen for human hepatocellular carcinoma," Journal of Onco Targets and Therapy, vol. 7, pp. 1061-1070, 2014.

[33] L. H. Butterfield, A. Ribas, W. S. Meng et al., "Tcell responses to HLA-A*0201 immunodominant peptides derived from alphafetoprotein in patients with hepatocellular cancer," Clinical Cancer Research, vol. 9, no. 16, part 1, pp. 5902-5908, 2003.

[34] H. Komori, T. Nakatsura, S. Senju et al., "Identification of HLA-A2- or HLA-A24-restricted CTL epitopes possibly useful for glypican-3-specific immunotherapy of hepatocellular carcinoma," Clinical Cancer Research, vol. 12, no. 9, pp. 2689-2697, 2006.

[35] H. Xu, N. Gu, Z.-B. Liu et al., "NY-ESO-1 expression in hepatocellular carcinoma: a potential new marker for early recurrence after surgery," Oncology Letters, vol. 3, no. 1, pp. 39-44, 2012. 
[36] F. Korangy, L. A. Ormandy, J. S. Bleck et al., "Spontaneous tumor-specific humoral and cellular immune responses to NYESO-1 in hepatocellular carcinoma," Clinical Cancer Research, vol. 10, no. 13, pp. 4332-4341, 2004.

[37] G. Bricard, H. Bouzourene, O. Martinet et al., "Naturally acquired MAGE-A10- and SSX-2-specific CD8 ${ }^{+} \mathrm{T}$ cell responses in patients with hepatocellular carcinoma," The Journal of Immunology, vol. 174, no. 3, pp. 1709-1716, 2005.

[38] J. Liang, T. Ding, Z.-W. Guo et al., "Expression pattern of tumour-associated antigens in hepatocellular carcinoma: association with immune infiltration and disease progression," British Journal of Cancer, vol. 109, no. 4, pp. 1031-1039, 2013.

[39] A. Zerbini, M. Pilli, P. Soliani et al., "Ex vivo characterization of tumor-derived melanoma antigen encoding gene-specific CD8+ cells in patients with hepatocellular carcinoma," Journal of Hepatology, vol. 40, no. 1, pp. 102-109, 2004.

[40] L. Carulli and C. Anzivino, "Telomere and telomerase in chronic liver disease and hepatocarcinoma," World Journal of Gastroenterology, vol. 20, no. 20, pp. 6287-6292, 2014.

[41] A. W. Bird and A. A. Hyman, "Building a spindle of the correct length in human cells requires the interaction between TPX2 and Aurora A," The Journal of Cell Biology, vol. 182, no. 2, pp. 289-300, 2008.

[42] S. L. Warner, B. J. Stephens, S. Nwokenkwo et al., "Validation of TPX2 as a potential therapeutic target in pancreatic cancer cells," Clinical Cancer Research, vol. 15, no. 21, pp. 6519-6528, 2009.

[43] Y. Ma, D. Lin, W. Sun et al., "Expression of targeting protein for Xklp2 associated with both malignant transformation of respiratory epithelium and progression of squamous cell lung cancer," Clinical Cancer Research, vol. 12, no. 4, pp. 1121-1127, 2006.

[44] S. Tanaka, S. Arii, M. Yasen et al., "Aurora kinase B is a predictive factor for the aggressive recurrence of hepatocellular carcinoma after curative hepatectomy," British Journal of Surgery, vol. 95, no. 5, pp. 611-619, 2008.

[45] C. W. Yang, J. Y. Su, A. P. Tsou et al., "Integrative genomics based identification of potential human hepatocarcinogenesisassociated cell cycle regulators: RHAMM as an example," Biochemical and Biophysical Research Communications, vol. 330, no. 2, pp. 489-497, 2005.

[46] R. Satow, M. Shitashige, Y. Kanai et al., "Combined functional genome survey of therapeutic targets for hepatocellular carcinoma," Clinical Cancer Research, vol. 16, no. 9, pp. 2518-2528, 2010.

[47] M. A. Burchill, J. Yang, K. B. Vang, and M. A. Farrar, "Interleukin-2 receptor signaling in regulatory $\mathrm{T}$ cell development and homeostasis," Immunology Letters, vol. 114, no. 1, pp. 1-8, 2007.

[48] A. M. Thornton and E. M. Shevach, " $\mathrm{CD} 4{ }^{+} \mathrm{CD} 25^{+}$immunoregulatory $\mathrm{T}$ cells suppress polyclonal $\mathrm{T}$ cell activation in vitro by inhibiting interleukin 2 production," The Journal of Experimental Medicine, vol. 188, no. 2, pp. 287-296, 1998.

[49] C. A. Piccirillo and E. M. Shevach, "Cutting edge: control of $\mathrm{CD} 8+\mathrm{T}$ cell activation by $\mathrm{CD} 4^{+} \mathrm{CD} 25^{+}$immunoregulatory cells," The Journal of Immunology, vol. 167, no. 3, pp. 1137-1140, 2001.

[50] R. S. Bystry, V. Aluvihare, K. A. Welch, M. Kallikourdis, and A. G. Betz, "B cells and professional APCs recruit regulatory T cells via CCL4," Nature Immunology, vol. 2, no. 12, pp. 1126-1132, 2001.
[51] S. Onizuka, I. Tawara, J. Shimizu, S. Sakaguchi, T. Fujita, and E. Nakayama, "Tumor rejection by in vivo administration of antiCD25 (interleukin-2 receptor $\alpha$ ) monoclonal antibody," Cancer Research, vol. 59, no. 13, pp. 3128-3133, 1999.

[52] A. Gallimore and S. Sakaguchi, "Regulation of tumour immunity by CD25 ${ }^{+}$T cells," Immunology, vol. 107, no. 1, pp. 5-9, 2002.

[53] E. M. Aandahl, J. Michaëlsson, W. J. Moretto, F. M. Hecht, and D. F. Nixon, "Human $\mathrm{CD}^{+} \mathrm{CD}^{+} 5^{+}$regulatory $\mathrm{T}$ cells control T-cell responses to human immunodeficiency virus and cytomegalovirus antigens," Journal of Virology, vol. 78, no. 5, pp. 2454-2459, 2004.

[54] E. Unitt, S. M. Rushbrook, A. Marshall et al., "Compromised lymphocytes infiltrate hepatocellular carcinoma: the role of Tregulatory cells," Hepatology, vol. 41, no. 4, pp. 722-730, 2005.

[55] H. Wu, P. Chen, R. Liao et al., "Intratumoral regulatory T cells with higher prevalence and more suppressive activity in hepatocellular carcinoma patients," Journal of Gastroenterology and Hepatology, vol. 28, no. 9, pp. 1555-1564, 2013.

[56] H. Q. Zhao, W. M. Li, Z. Q. Lu, and Y. M. Yao, "Roles of tregs in development of hepatocellular carcinoma: a meta-analysis," World Journal of Gastroenterology, vol. 20, no. 24, pp. 7971-7978, 2014.

[57] C. Miroux, T. Vausselin, and N. Delhem, "Regulatory T cells in $\mathrm{HBV}$ and HCV liver diseases: implication of regulatory T lymphocytes in the control of immune response," Expert Opinion on Biological Therapy, vol. 10, no. 11, pp. 1563-1572, 2010.

[58] J. Schrader, "The role of MDSCs in hepatocellular carcinoma: in vivo veritas?” Journal of Hepatology, vol. 59, no. 5, pp. 921-923, 2013.

[59] C.-E. Hu, J. Gan, R.-D. Zhang, Y.-R. Cheng, and G.-J. Huang, "Up-regulated myeloid-derived suppressor cell contributes to hepatocellular carcinoma development by impairing dendritic cell function," Scandinavian Journal of Gastroenterology, vol. 46, no. 2, pp. 156-164, 2011.

[60] P. Sinha, V. K. Clements, S. K. Bunt, S. M. Albelda, and S. Ostrand-Rosenberg, "Cross-talk between myeloid-derived suppressor cells and macrophages subverts tumor immunity toward a type 2 response," The Journal of Immunology, vol. 179, no. 2, pp. 977-983, 2007.

[61] D. Ilkovitch and D. M. Lopez, "The liver is a site for tumorinduced myeloid-derived suppressor cell accumulation and immunosuppression," Cancer Research, vol. 69, no. 13, pp. 55145521, 2009.

[62] D. Gabrilovich, "Mechanisms and functional significance of tumour-induced dendritic-cell defects," Nature Reviews Immunology, vol. 4, no. 12, pp. 941-952, 2004.

[63] B. Hoechst, T. Voigtlaender, L. Ormandy et al., "Myeloid derived suppressor cells inhibit natural killer cells in patients with hepatocellular carcinoma via the NKP30 receptor," Hepatology, vol. 50, no. 3, pp. 799-807, 2009.

[64] B. Hoechst, L. A. Ormandy, M. Ballmaier et al., "A new population of myeloid-derived suppressor cells in hepatocellular carcinoma patients induces CD $4^{+} \mathrm{CD} 25^{+}$Foxp $3^{+} \mathrm{T}$ cells," Gastroenterology, vol. 135, no. 1, pp. 234-243, 2008.

[65] B. Hoechst, T. Voigtlaender, L. Ormandy et al., "Myeloid derived suppressor cells inhibit natural killer cells in patients with hepatocellular carcinoma via the NKp30 receptor," Hepatology, vol. 50, no. 3, pp. 799-807, 2009.

[66] F. Arihara, E. Mizukoshi, M. Kitahara et al., "Increase in CD14 ${ }^{+} \mathrm{HLA}_{-} \mathrm{DR}^{-/ \text {low }}$ myeloid-derived suppressor cells in hepatocellular carcinoma patients and its impact on prognosis," 
Cancer Immunology, Immunotherapy, vol. 62, no. 8, pp. 14211430, 2013.

[67] T. F. Greten, L. A. Ormandy, A. Fikuart et al., "Low-dose cyclophosphamide treatment impairs regulatory $\mathrm{T}$ cells and unmasks AFP-specific CD4+ T-cell responses in patients with advanced HCC," Journal of Immunotherapy, vol. 33, no. 2, pp. 211-218, 2010.

[68] Y. Han, Z. Chen, Y. Yang et al., "Human CD $14^{+}$CTLA- $4^{+}$regulatory dendritic cells suppress T-cell response by cytotoxic Tlymphocyte antigen-4-dependent IL-10 and indoleamine-2,3dioxygenase production in hepatocellular carcinoma," Hepatology, vol. 59, no. 2, pp. 567-579, 2014.

[69] T. Hato, L. Goyal, T. F. Greten, D. G. Duda, and A. X. Zhu, "Immune checkpoint blockade in hepatocellular carcinoma: current progress and future directions," Hepatology, vol. 60, no. 5, pp. 1776-1782, 2014.

[70] B. Hoechst, L. A. Ormandy, M. Ballmaier et al., "A new population of myeloid-derived suppressor cells in hepatocellular carcinoma patients induces CD $4^{+} \mathrm{CD} 25^{+}$Foxp ${ }^{+}$T Cells," Gastroenterology, vol. 135, no. 1, pp. 234-243, 2008.

[71] K. Fujiwara, T. Higashi, K. Nouso et al., "Decreased expression of B7 costimulatory molecules and major histocompatibility complex class-I in human hepatocellular carcinoma," Journal of Gastroenterology and Hepatology, vol. 19, no. 10, pp. 1121-1127, 2004.

[72] K. Kurokohchi, M. Carrington, D. L. Mann et al., "Expression of HLA class I molecules and the transporter associated with antigen processing in hepatocellular carcinoma," Hepatology, vol. 23, no. 5, pp. 1181-1188, 1996.

[73] T. Tatsumi, T. Takehara, K. Katayama et al., "Expression of costimulatory molecules B7-1 (CD80) and B7-2 (CD86) on human hepatocellular carcinoma," Hepatology, vol. 25, no. 5, pp. 1108-1114, 1997.

[74] F. Shi, M. Shi, Z. Zeng et al., "PD-1 and PD-L1 upregulation promotes $\mathrm{CD}^{+}$T-cell apoptosis and postoperative recurrence in hepatocellular carcinoma patients," International Journal of Cancer, vol. 128, no. 4, pp. 887-896, 2011.

[75] S. Chen, L.-F. Lee, T. S. Fisher et al., "Combination of 4-1BB agonist and $\mathrm{PD}-1$ antagonist promotes anti-tumor effector/memory CD8 T cells in a poorly immunogenic tumor model," Cancer Immunology Research, 2014.

[76] R. S. Herbst, J. C. Soria, M. Kowanetz et al., "Predictive correlates of response to the anti-PD-L1 antibody MPDL3280A in cancer patients," Nature, vol. 515, no. 7528, pp. 563-567, 2014.

[77] B. S. Henick, R. S. Herbst, and S. B. Goldberg, "The PD-1 pathway as a therapeutic target to overcome immune escape mechanisms in cancer," Expert Opinion on Therapeutic Targets, vol. 18, no. 12, pp. 1407-1420, 2014.

[78] D. Petrovic, Z. Stamataki, E. Dempsey et al., "Hepatitis C virus targets the $\mathrm{T}$ cell secretory machinery as a mechanism of immune evasion," Hepatology, vol. 53, no. 6, pp. 1846-1853, 2011.

[79] J. M. O’Bryan, J. A. Potts, H. L. Bonkovsky, A. Mathew, and A. L. Rothman, "Extended interferon-alpha therapy accelerates telomere length loss in human peripheral blood T lymphocytes," PLoS ONE, vol. 6, no. 8, Article ID e20922, 2011.

[80] A. D. Pardee and L. H. Butterfield, "Immunotherapy of hepatocellular carcinoma: unique challenges and clinical opportunities," OncoImmunology, vol. 1, no. 1, pp. 48-55, 2012.

[81] T. Stroffolini, A. Spadaro, V. Di Marco et al., "Current practice of chronic hepatitis B treatment in Southern Italy," European Journal of Internal Medicine, vol. 23, no. 5, pp. e124-e127, 2012.
[82] G. Bertino, A. Ardiri, P. M. Boemi et al., "Epoetin alpha improves the response to antiviral treatment in HCV-related chronic hepatitis," European Journal of Clinical Pharmacology, vol. 66, no. 10, pp. 1055-1063, 2010.

[83] G. Malaguarnera, M. Pennisi, C. Gagliano et al., "Acetyl-Lcarnitine supplementation during HCV therapy with pegylated interferon- $\alpha 2 \mathrm{~b}$ plus ribavirin: effect on work performance; a randomized clinical trial," Hepatitis Monthly, vol. 14, no. 5, Article ID e11608, 2014.

[84] M. Malaguarnera, M. Vacante, M. Giordano et al., "L-carnitine supplementation improves hematological pattern in patients affected by HCV treated with peg interferon- $\alpha 2 \mathrm{~b}$ plus ribavirin," World Journal of Gastroenterology, vol. 17, no. 39, pp. 4414-4420, 2011.

[85] M. Malaguarnera, M. Vacante, G. Bertino et al., "The supplementation of acetyl-L-carnitine decreases fatigue and increases quality of life in patients with hepatitis $C$ treated with pegylated interferon- $\alpha$ 2b plus ribavirin," Journal of Interferon and Cytokine Research, vol. 31, no. 9, pp. 653-659, 2011.

[86] C. M. Bruno, M. Valenti, G. Bertino et al., "Relationship between circulating interleukin-10 and histological features in patients with chronic C hepatitis," Annals of Saudi Medicine, vol. 31, no. 4, pp. 360-364, 2011.

[87] F. Zhao, F. Korangy, and T. F. Greten, "Cellular immune suppressor mechanisms in patients with hepatocellular carcinoma," Digestive Diseases, vol. 30, no. 5, pp. 477-482, 2012.

[88] L. Cai, Z. Zhang, L. Zhou et al., "Functional impairment in circulating and intrahepatic NK cells and relative mechanism in hepatocellular carcinoma patients," Clinical Immunology, vol. 129, no. 3, pp. 428-437, 2008.

[89] M. El Ansary, S. Mogawer, S. A. Elhamid et al., "Immunotherapy by autologous dendritic cell vaccine in patients with advanced HCC," Journal of Cancer Research and Clinical Oncology, vol. 139, no. 1, pp. 39-48, 2013.

[90] F. Zhao, F. Korangy, and T. F. Greten, "Cellular immune suppressor mechanisms in patients with hepatocellular carcinoma," Digestive Diseases, vol. 30, no. 5, pp. 477-482, 2012.

[91] J.-H. Zhong, L. Ma, L.-C. Wu et al., "Adoptive immunotherapy for postoperative hepatocellular carcinoma: a systematic review," International Journal of Clinical Practice, vol. 66, no. 1, pp. 21-27, 2012.

[92] W. C. Lee, H. C. Wang, C. F. Hung, P. F. Huang, C. R. Lia, and M. F. Chen, "Vaccination of advanced hepatocellular carcinoma patients with tumor lysate-pulsed dendritic cells: a clinical trial," Journal of Immunotherapy, vol. 28, no. 5, pp. 496-504, 2005.

[93] E. Mizukoshi, Y. Nakamoto, K. Arai et al., "Comparative analysis of various tumor-associated antigen-specific $t$-cell responses in patients with hepatocellular carcinoma," Hepatology, vol. 53, no. 4, pp. 1206-1216, 2011.

[94] F. S. Hodi, S. J. O'Day, D. F. McDermott et al., "Improved survival with ipilimumab in patients with metastatic melanoma," The New England Journal of Medicine, vol. 363, no. 8, pp. 711723, 2010.

[95] C. Robert, L. Thomas, I. Bondarenko et al., "Ipilimumab plus dacarbazine for previously untreated metastatic melanoma," The New England Journal of Medicine, vol. 364, no. 26, pp. 25172526, 2011.

[96] L. Calabrò, A. Morra, E. Fonsatti et al., "Tremelimumab for patients with chemotherapy-resistant advanced malignant mesothelioma: an open-label, single-arm, phase 2 trial," The Lancet Oncology, vol. 14, no. 11, pp. 1104-1111, 2013. 
[97] B. Sangro, C. Gomez-Martin, M. de la Mata et al., "A clinical trial of CTLA- 4 blockade with tremelimumab in patients with hepatocellular carcinoma and chronic hepatitis C,' Journal of Hepatology, vol. 59, no. 1, pp. 81-88, 2013.

[98] J. F. Grosso and M. N. Jure-Kunkel, "CTLA-4 blockade in tumor models: an overview of preclinical and translational research," Cancer immunity, vol. 13, pp. 5-19, 2013.

[99] K. Sakuishi, L. Apetoh, J. M. Sullivan, B. R. Blazar, V. K. Kuchroo, and A. C. Anderson, "Targeting Tim-3 and PD-1 pathways to reverse $\mathrm{T}$ cell exhaustion and restore anti-tumor immunity," The Journal of Experimental Medicine, vol. 207, no. 10, pp. 2187-2194, 2010.

[100] O. Hamid, C. Robert, A. Daud et al., "Safety and tumor responses with lambrolizumab (anti-PD-1) in melanoma," The New England Journal of Medicine, vol. 369, no. 2, pp. 134-144, 2013.

[101] J. D. Wolchok, H. Kluger, M. K. Callahan et al., "Nivolumab plus Ipilimumab in advanced melanoma," The New England Journal of Medicine, vol. 369, no. 2, pp. 122-133, 2013.

[102] R. Berger, R. Rotem-Yehudar, G. Slama et al., "Phase i safety and pharmacokinetic study of CT-011, a humanized antibody interacting with PD-1, in patients with advanced hematologic malignancies," Clinical Cancer Research, vol. 14, no. 10, pp. 3044-3051, 2008.

[103] A. D. Pardee, D. McCurry, S. Alber, P. Hu, A. L. Epstein, and W. J. Storkus, "A therapeutic OX40 agonist dynamically alters dendritic, endothelial, and $\mathrm{T}$ cell subsets within the established tumor microenvironment," Cancer Research, vol. 70, no. 22, pp. 9041-9052, 2010.

[104] J. M. Llovet, S. Ricci, V. Mazzaferro et al., "Sorafenib in advanced hepatocellular carcinoma," The New England Journal of Medicine, vol. 359, no. 4, pp. 378-390, 2008.

[105] B. Xie, D. H. Wang, and S. J. Spechler, "Sorafenib for treatment of hepatocellular carcinoma: a systematic review," Digestive Diseases and Sciences, vol. 57, no. 5, pp. 1122-1129, 2012.

[106] A. X. Zhu, D. G. Duda, D. V. Sahani, and R. K. Jain, "HCC and angiogenesis: Possible targets and future directions," Nature Reviews Clinical Oncology, vol. 8, no. 5, pp. 292-301, 2011.

[107] J. Heo, T. Reid, L. Ruo et al., "Randomized dose-finding clinical trial of oncolytic immunotherapeutic vaccinia JX-594 in liver cancer," Nature Medicine, vol. 19, no. 3, pp. 329-336, 2013.

[108] L. Liu, W. Wang, H. Chen et al., "EASL- and mRECISTevaluated responses to combination therapy predict survival in patients with hepatocellular carcinoma," Clinical Cancer Research, vol. 20, no. 6, pp. 1623-1631, 2014.

[109] F. Y. Yao, "Conundrum of treatment for early-stage hepatocellular carcinoma: radiofrequency ablation instead of liver transplantation as the first-line treatment?" Liver Transplantation, vol. 20, no. 3, pp. 257-260, 2014.

[110] N. Mehta, J. L. Dodge, J. P. Roberts, R. Hirose, and F. Y. Yao, "Outcomes after liver transplantation for patients with hepatocellular carcinoma and a low risk of dropout from the transplant waiting list," Liver Transplantation, vol. 20, no. 5, pp. 627-628, 2014.

[111] J. M. Llovet and J. Bruix, "Molecular targeted therapies in hepatocellular carcinoma," Hepatology, vol. 48, no. 4, pp. 1312-1327, 2008.

[112] M. Y. Ali, C. F. Grimm, M. Ritter et al., "Activation of dendritic cells by local ablation of hepatocellular carcinoma," Journal of Hepatology, vol. 43, no. 5, pp. 817-822, 2005.
[113] R. L. Bogorad, H. Yin, A. Zeigerer et al., "Nanoparticle-formulated siRNA targeting integrins inhibits hepatocellular carcinoma progression in mice," Nature Communications, vol. 5, article 3869, 2014.

[114] X. H. Huang, W. H. Jian, Z. F. Wu et al., "Small interfering RNA (siRNA)-mediated knockdown of macrophage migration inhibitory factor (MIF) suppressed cyclin D1 expression and hepatocellular carcinoma cell proliferation," Oncotarget, vol. 5, no. 14, pp. 5570-5580, 2014.

[115] G. K. Abou-Alfa, "Sorafenib use in hepatocellular carcinoma: more questions than answers," Hepatology, vol. 60, no. 1, pp. 1518, 2014.

[116] T. Arizumi, K. Ueshima, H. Takeda et al., "Comparison of systems for assessment of post-therapeutic response to sorafenib for hepatocellular carcinoma," Journal of Gastroenterology, vol. 49, no. 12, pp. 1578-1587, 2014.

[117] G. Bertino, I. Di Carlo, A. Ardiri et al., "Systemic therapies in hepatocellular carcinoma: present and future," Future Oncology, vol. 9, no. 10, pp. 1533-1548, 2013.

[118] G. Bertino, S. Demma, N. Bertino, and A. Ardiri, "Management of hepatocellular carcinoma: an update at the start of 2014," Journal of Gastrointestinal \& Digestive System, vol. 4, no. 2, pp. $1-7,2014$.

[119] G. Bertino, S. Demma, A. Ardiri et al., "Hepatocellular carcinoma: novel molecular targets in carcinogenesis for future therapies," BioMed Research International, vol. 2014, Article ID 203693, 15 pages, 2014. 